\title{
BMJ Open Motor imagery ability assessments in four disciplines: protocol for a systematic review
}

\author{
Zorica Suica, ${ }^{1}$ Petra Platteau-Waldmeier, ${ }^{2}$ Szabina Koppel, ${ }^{1}$ \\ Arno Schmidt-Trucksaess, ${ }^{3}$ Thierry Ettlin, ${ }^{1}$ Corina Schuster-Amft ${ }^{1,3,4}$
}

To cite: Suica Z, PlatteauWaldmeier P, Koppel S et al. Motor imagery ability assessments in four disciplines: protocol for a systematic review. BMJ Open 2018;8:e023439. doi:10.1136/ bmjopen-2018-023439

- Prepublication history for this paper is available online. To view these files, please visit the journal online (http://dx.doi. org/10.1136/bmjopen-2018023439).

Received 9 April 2018 Revised 28 August 2018 Accepted 17 October 2018

A) Check for updates

(c) Author(s) (or their employer(s)) 2018. Re-use permitted under CC BY-NC. No commercial re-use. See rights and permissions. Published by BMJ.

${ }^{1}$ Research Department, Reha Rheinfelden, Rheinfelden,

Switzerland

${ }^{2}$ School of Health Professions, Institute of Physiotherapy, Zurich University for Applied Sciences, Winterthur, Switzerland

${ }^{3}$ Department for Sport, Exercise and Health, University of Basel, Basel, Switzerland

${ }^{4}$ Institute for Rehabilitation and Performance Technology, Bern University of Applied Sciences, Burgdorf, Switzerland

Correspondence to Dr Corina Schuster-Amft; c.schuster@reha-rhf.ch

\section{ABSTRACT}

Introduction Motor imagery (MI) is a very popular and well-accepted technique in different disciplines. Originating from sport and psychology, $\mathrm{Ml}$ is now also used in the field of medicine and education. Several studies confirmed the benefits of MI to facilitate motor learning and skill acquisition. The findings indicated that individual's $\mathrm{Ml}$ ability might influence the effectiveness of Ml interventions. Over the last two centuries, researchers have developed several assessments to evaluate Ml's abstract construct. However, no systematic reviews (SR) exist for Ml ability evaluation methods and their measurement properties.

Methods and analysis The SR will evaluate available $\mathrm{Ml}$ ability assessments and their psychometric properties in four relevant disciplines: sports, psychology, medicine and education. This involves performing searches in SPORTDiscus, PsycINFO, Cochrane Library, Scopus, Web of Science and ERIC. Working independently, two reviewers will screen articles for selection. Then all raw information will be compiled in an overview table-including the articles' characteristics (eg, a study's setting or the population demographics) and the Ml ability assessment (psychometric properties). To evaluate the articles' methodological quality, we will use the COSMIN checklist. Then we will evaluate all the included assessments' quality and perform a best-evidence synthesis. Results of this review will be reported following the Preferred Reporting Items for Systematic Reviews and Meta-Analyses (PRISMA) guidelines.

Ethics and dissemination The SR is based on published data, and ethical approval is not required. This review will provide information on assessment performance and equipment, as well as its main focus and usefulness. Furthermore, we will present the methodological quality of all the included articles and assess the included instruments' quality. Ultimately, this will act as a valuable resource, providing an overview of Ml ability assessments for individual clinical settings, treatment aims, and various populations. The SR's final report will be published in a peer-reviewed journal and presented at relevant conferences.

PROSPERO registration number CRD42017077004

\section{INTRODUCTION}

Motor imagery (MI) - the mental representation of an action without engaging in its

\section{Strengths and limitations of this study}

- The systematic review will provide an overview of available assessments of Motor imagery (MI) ability in the following disciplines: sports, psychology, medicine and education.

- The included articles' methodological quality will be assessed and reported using the Consensus-based Standards for the selection of health Measurement Instruments guidelines.

- The selected MI ability assessments' quality will be described.

- The review will not be restricted to a specific population, age or disease.

- The review will be restricted to literature published in English and German languages.

physical execution-is a widely used technique in many different disciplines (eg, sports, psychology, medicine and education). ${ }^{12}$ The technique of MI has been shown to substantially enhance motor rehabilitation in patients following: stroke, ${ }^{34}$ spinal cord injury, ${ }^{5}$ orthopaedic surgeries ${ }^{6-8}$ and sport injuries. ${ }^{910}$ Furthermore, MI is a very popular strategy to enhance psychomotor skills 11-13 or various aspects of performance among athletes $^{14}$.

MI can be explicit or implicit. Explicit MI is the voluntary active imagination of a movement with conscious mental representation. ${ }^{15-17}$ Determining the laterality of a picture of a human hand refers to a mental rotation task. The mental rotation task requires individuals to mentally rotate an internal representation of their own body part into the presented part's position. Unconscious imagining of movement during a mental rotation task is considered implicit MI. Imagined movements can be stimulated mentally, using either the kinaesthetic (sensation of movement) or the visual mode (visualisation of movement) $^{18}$ and can be viewed from an internal or external perspective. ${ }^{19}$ By using the internal perspective, 
Table 1 Overview of inclusion criteria

\begin{tabular}{ll}
\hline Inclusion criteria & \\
\hline Construct & $\begin{array}{l}\text { Motor imagery, mental imagery, mental rehearsal, movement imagery, mental practice, mental training, } \\
\text { mental simulation and visualisation. }\end{array}$ \\
Field of interest & Sports, psychology, medicine and education. \\
\hline Target population & $\begin{array}{l}\text { Not limited to a specific population: ie, healthy individuals, adults, children and patients. No restriction on } \\
\text { age, gender or health status. }\end{array}$ \\
Assessment instrument & $\begin{array}{l}\text { All assessment methods, standardised assessments or questionnaires, rating tests, congruency tests and a } \\
\text { mental chronometry test without limitations on a version or language. }\end{array}$ \\
Measurement properties & $\begin{array}{l}\text { Reliability parameters: internal consistency, measurement errors, test-retest reliability, inter-rater and } \\
\text { intrarater reliability. } \\
\text { Validity parameters: content, construct and criterion validity. } \\
\text { Responsiveness parameters: SE of measurement, minimal detectable change and standardised response } \\
\text { mean . }\end{array}$ \\
Publication language & English and German. \\
\hline
\end{tabular}

the movement is imagined from a first-person view, for example, as though viewing through one's own eyes the body part move. Contrarily, when using the external perspective, it is a third-person view of oneself, similar to an observer watching a movement execution on television. ${ }^{19} 20$

Several neuroimaging studies have shown that the brain areas active during MI are similar to those active during the actual movement. ${ }^{21-23}$ Furthermore, recent findings showed that MI activates subcortical structures (ie, the excitability of presynaptic interneurons) without activating alpha-motoneurons. ${ }^{24}$ This led to the theory that MI facilitates motor activity and excite the movement execution, eventually improving motor function or performance, for example, movement accuracy, gait speed or strength. 3472526

However, measuring MI ability is no simple feat because MI is actually a multidimensional construct ${ }^{27}$ with wide individual differences. ${ }^{28} 29$

Martin et $a l^{30}$ showed that an individual's MI ability can influence his/her effectiveness to achieve intended outcomes. Therefore, it is deemed essential to assess MI abilities prior to $\mathrm{MI}$ interventions. ${ }^{14}$

Over the last two centuries, various assessments were developed to evaluate an individual's MI ability within different dimensions, for example, vividness or image clarity $^{31-39}$ and controllability or the ease and accuracy with which an image can be manipulated mentally. ${ }^{40-43}$ Some assessments can evaluate both dimensions - vividness and controllability. ${ }^{44}$ However, all these assessments are often used as self-reported questionnaires for subjective MI ability assessments. $^{245}$

Objective assessment methods, on the other hand, record central and peripheral nervous system activities during MI. These methods could be categorised as neurophysiological methods-for example, functional MRI (fMRI), positron emission tomography, electromyography, electro-oculography and electroencephalography (EEG). ${ }^{2}$

Although MI ability assessments are easy to use and cost-effective, ${ }^{45}$ they do not allow control of MI ability before or during a clinical experiment. ${ }^{2}$ Objective assessment methods are more powerful and versatile assessments of the MI duration and the temporal congruence between imagery and the time taken to execute the same movements ${ }^{45}$ but the assessments cannot evaluate MI qualities, such as the MI perspective, vividness, ease and so forth. ${ }^{2}$

Thus, no single assessment can determine an individual's ability or disability to perform MI. ${ }^{46}$ So, for a comprehensive evaluation of MI ability, including different aspects of MI, a combination of MI ability assessments is recommended. ${ }^{27} 4546$ This could include evaluating mental rotation, the temporal congruency of the same movement's mental and practical performance, for example, the Chaotic Motor Imagery Assessment, and a standardised questionnaire such as the Kinaesthetic and Visual Imagery Questionnaire or Movement Imagery Questionnaire. $^{47}$

However, the literature is still remiss in providing a systematic review of MI evaluation methods and their measurement properties. ${ }^{2}$ For example, Di Rienzo et $a l$, McAvinue and Robertson, and Melogno-Klinkas et al mainly focused on evaluating the MI ability in the field of neurology for healthy individuals or athletes. ${ }^{2} 1748$ Only two of those reviews reported the assessments' psychometric properties. ${ }^{17}{ }^{48}$ Furthermore, Melogno-Klinkas et $a l^{48}$ only included assessments designed for a Spanish-speaking community in neurorehabilitation. McAvinue et $a l^{17}$ summarised self-reported questionnaires for measuring explicit MI restricted to sport and exercise. The review by Di Rienzo et al ${ }^{2}$ provided an overview on assessments used for MI ability evaluation but it was limited to the field of neurorehabilitation-for example, patients after stroke, with Parkinson's disease or spinal cord injury and patients after an amputation in clinical settings.

In contrast, our proposed systematic review will enable clinicians, coaches, teachers and researchers to select a suitable MI ability assessment for their current settings and goals based on information provided regarding the assessment's focus and quality. Essentially, the review will 
Table 2 Search strategy

\begin{tabular}{|c|c|}
\hline Search domain & Search words \\
\hline \multirow[t]{2}{*}{ 1. construct } & $\begin{array}{l}\text { 'motor imagery' OR 'mental imagery' OR 'mental rehearsal' OR 'movement imagery' OR 'mental } \\
\text { practice' OR 'mental training' }\end{array}$ \\
\hline & AND \\
\hline \multirow[t]{2}{*}{ 2. instrument } & 'Instrument' OR 'measurement' OR 'questionnaire' OR 'scale' OR 'assessment' OR 'test' \\
\hline & AND \\
\hline $\begin{array}{l}\text { 3. filter for measurement properties by } \\
\text { Terwee et al. }{ }^{51}\end{array}$ & 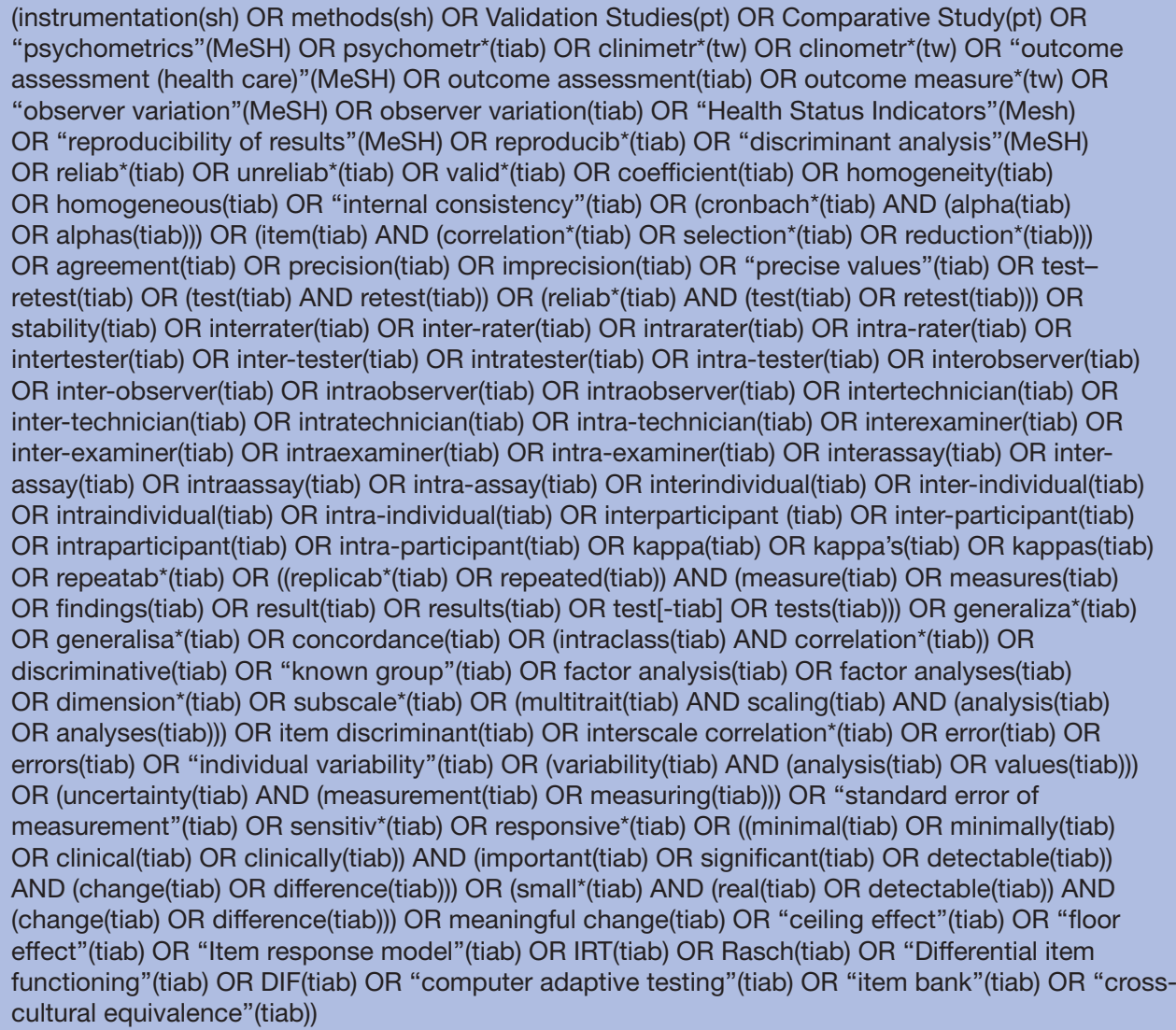 \\
\hline
\end{tabular}

IRT, item response theory; MeSH,medical subject headings; pt, publication type; sh, subheadings; tw, text word; tiab,title/abstract.

answer the question: What evaluated MI ability assessments are available in the fields of sports, psychology, medicine and education and what are their psychometric properties? Because of its enhanced utility, the review will provide an overview of the following:

1. Evaluated MI ability assessments, based on a systematic search-and-selection process, using either explicit or implicit MI in the fields of sports, psychology, medicine and education.

2. Current levels of evidence for psychometric properties of the selected MI ability assessments.

3. Necessary equipment and training for the included MI ability assessments.

\section{METHODS AND ANALYSIS}

\section{Study design and registration}

The study protocol was written and reported using the Preferred Reporting Items for Systematic Reviews and Meta-Analyses Protocols (PRISMA-P) guidelines and the PRISMA checklist. ${ }^{49}$ For this systematic review, we will follow the recommendations for systematic reviews on measurement properties. ${ }^{50}$

\section{Selection criteria}

Table 1 details the selection criteria applied during the systematic selection process, based on title, abstract and full text.

Articles will be excluded if the authors only use neurophysiological methods to evaluate MI ability, for example, fMRI, EEG or brain-computer interface technology.

\section{Search strategy}

One author (ZS) and a life science librarian from a medical library will carry out the search strategy independently in the defined databases. The following electronic databases will be searched from their inception for relevant articles:

- Sports-SPORTDiscus (1892 to current date of search);

- Psychology-PsycINFO (1887 to current date of search); 


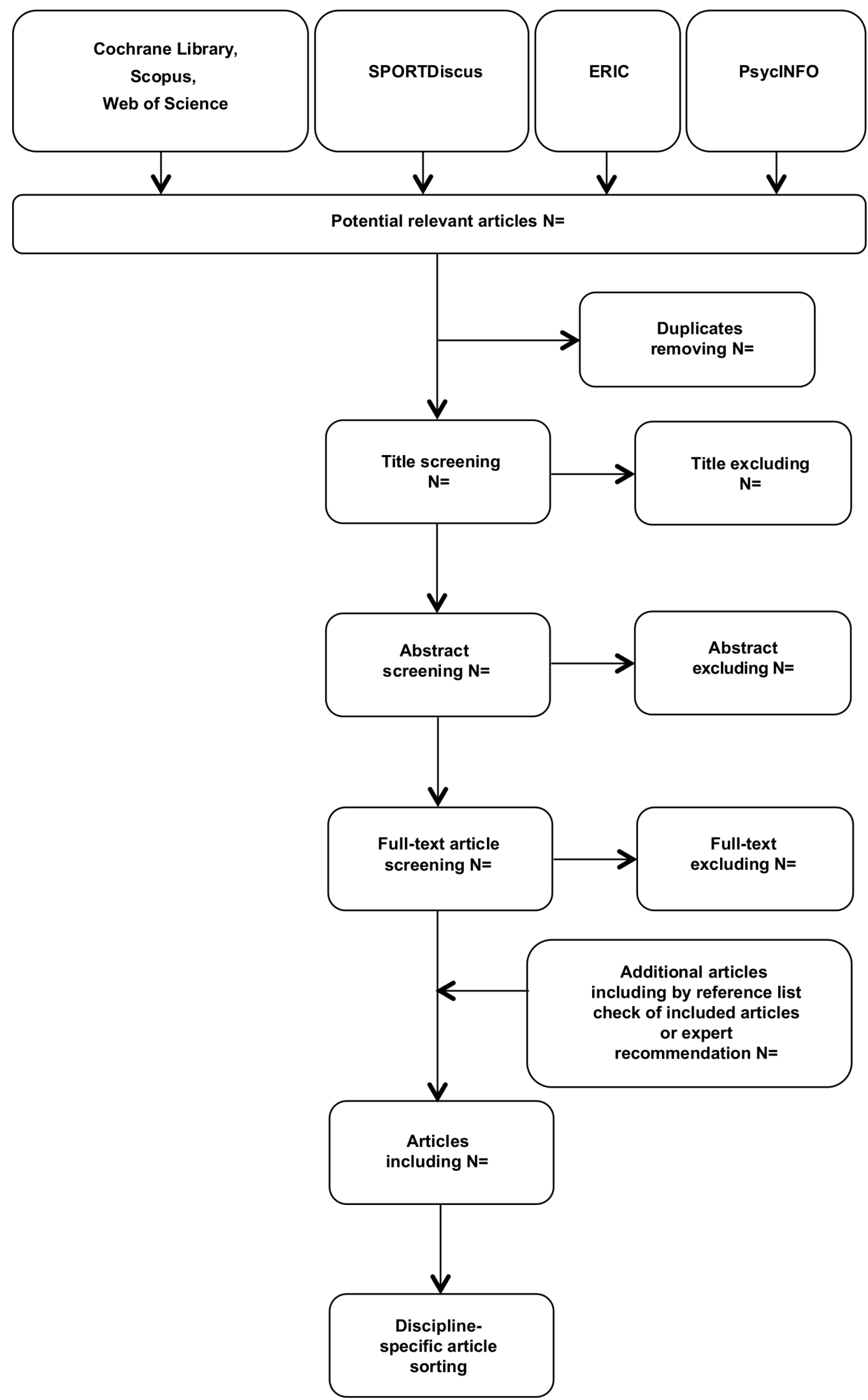

Figure 1 The literature selection process. $n=$ number of references.

- Medicine-Cochrane Library (current issue), Scopus (1996 to current date of search) and Web of Science (1900 to current date of search); and
Education-ERIC (1966 to current date of search). For each database, the search will include combined terms regarding the construct of interest and the 
Table 3 Quality criteria for measurement properties by Terwee et a/ ${ }^{55}$

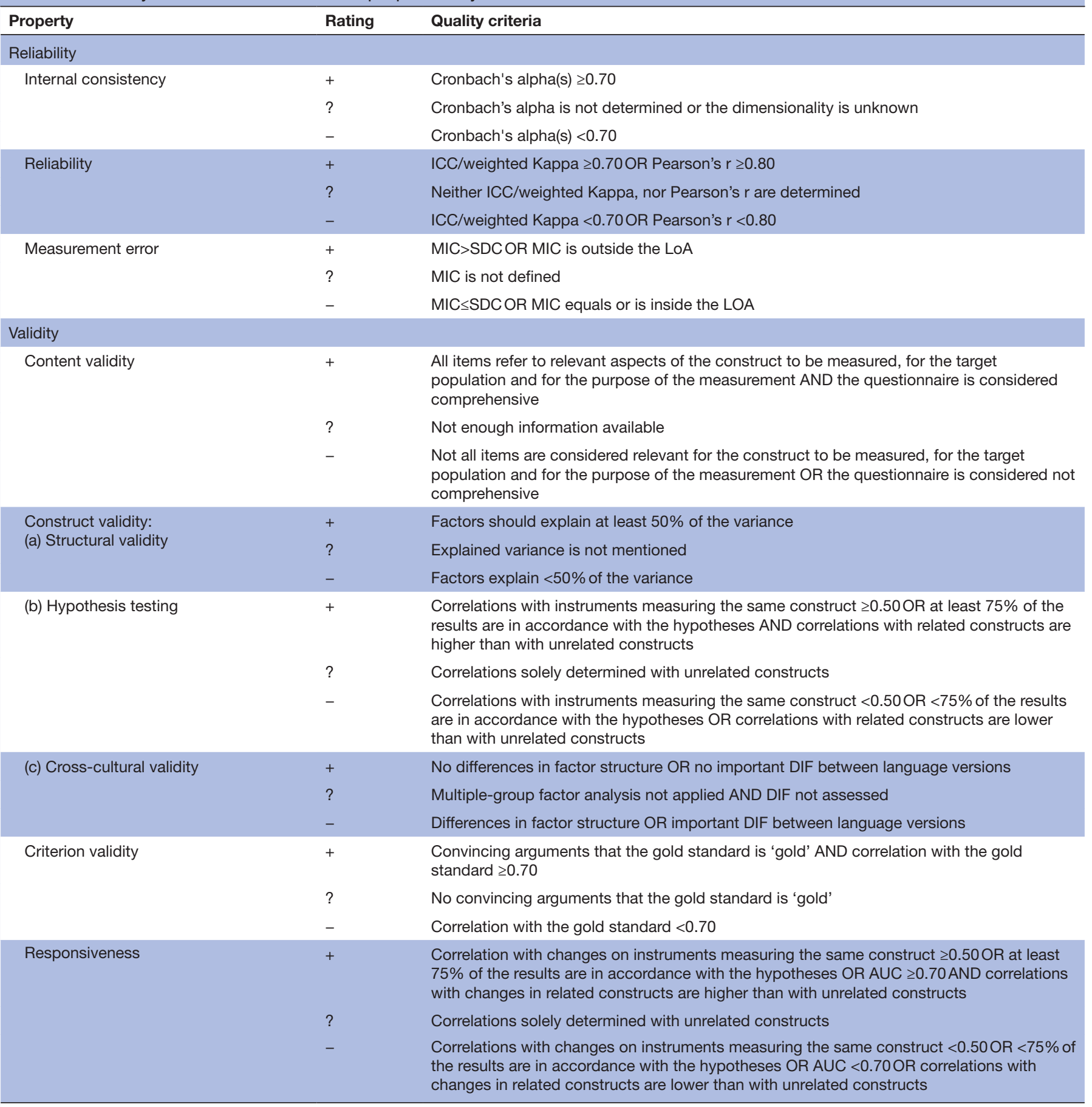

+, positive rating; -, negative rating.; ?, indeterminate rating; AUC, area under the curve; DIF, differential item functioning; ICC, intraclass correlation coefficient; LoA, limits of agreement; MIC, minimal important change; SDC, smallest detectable change.

assessment instrument. Furthermore, we will apply and adapt the search strategy proposed for each database (see table 2) to find articles using the Terwee $e t a l .^{51}$ measurement properties.

\section{Selection of studies}

We will upload, store and select the literature search results as figure 1 shows, with the help of a reference management software package, for example, EndNote (version X7; Thomson Reuters, NY, USA). The selection process will entail these steps:

1. For each database, we will create a separate library, while also keeping an original version.

2. For each library copy, we will create new libraries and subsequently merge them into one library.

3 . We will remove duplicates.

4. We will select publications based on their title and abstract first. 
Table 4 Levels of evidence for the overall quality of measurement properties from van Tulder et al ${ }^{56}$

\begin{tabular}{lll}
\hline Level & Rating & Criteria \\
\hline Strong & +++ or --- & $\begin{array}{l}\text { Consistent findings in } \\
\text { multiple studies of good } \\
\text { methodological quality } \\
\text { OR in one study of } \\
\text { excellent methodological } \\
\text { quality }\end{array}$ \\
Moderate & ++ or -- & $\begin{array}{l}\text { Consistent findings in } \\
\text { multiple studies of fair } \\
\text { methodological quality } \\
\text { OR in one study of good } \\
\text { methodological quality }\end{array}$ \\
& & $\begin{array}{l}\text { One study of fair } \\
\text { methodological quality }\end{array}$ \\
Limited & + or - & Conflicting findings \\
Conflicting & \pm & $\begin{array}{l}\text { Only studies of poor } \\
\text { methodological quality }\end{array}$ \\
\hline Unknown & $?$ &
\end{tabular}

+, positive rating; ?, indeterminate rating; -, negativerating.

5. To confirm final inclusion or exclusion, we will retrieve and screen the full text of the selected publications.

6. To find more potential articles, we will manually search the selected publications' reference lists.

7. We will classify the selected publications by discipline, for example, sports, psychology, medicine and education, based on the articles' focus and target population.

Two of the authors will work independently to screen all publications for inclusion or exclusion based on a customised selection sheet. We will note the reason for study exclusion in the reference record of the reference management software. In case of disagreement, we will try to reach consensus through discussion. If this should fail, we will consider the opinion of an independent third author. Selection congruency between the two independent reviewers will be measured using Cohen's Kappa. ${ }^{52}$

\section{Data extraction}

Two authors (CSA and ZS) will carry out the data extraction and a data extraction check independently, using a data extraction sheet specifically developed for this review. We will extract all raw information into Microsoft Excel (V.14.0, 2010, Microsoft, Redmond, California, USA). One researcher will extract data independently from all selected references. A second researcher will check the data extracted from the selected references.

Following de Vet et al. recommendation, ${ }^{50}$ we will extract the following information:

- Characteristics of included articles: first author, year of publication, country of origin, study design and the number and main characteristics of participants (eg, age, gender and target population).

- General characteristics of the assessment instrument: name; version; construct of evaluation; number of items; components of MI (kinaesthetic/visual mode or first-/third-person perspective) and subscales; scoring; assessment format; time and equipment needed; examiner qualifications; and costs.

- Data on the instruments' psychometric properties: validity, reliability and responsiveness.

If necessary, the included articles' authors will be contacted to obtain all the relevant information. A table providing an overview on data extraction will be created for each discipline separately.

\section{Outcomes}

The primary outcomes will be: (1) a description of available assessments of MI ability in the fields of sports, psychology, medicine and education; and (2) an evaluation of psychometric properties (the reliability, validity and responsiveness) of the selected MI ability assessments.

The secondary outcome will be to provide an overview of equipment and the training needed for all the selected assessments.

\section{Content comparison}

We will provide an overview of each assessment's content-covering the motor imagery's ability-using tables to visualise the similarities and differences among several MI ability assessments.

\section{Studies' methodological quality: the COSMIN evaluation}

To assess all the included articles' methodological quality, we will use the Consensus-based Standards for the selection of health Measurement Instruments (COSMIN) checklist published by Mokkink et al. ${ }^{53}$ The COSMIN checklist contains nine domains to assess the following measurement properties: internal consistency, reliability, measurement error, content validity, construct validity (eg, structural validity, hypotheses testing and cross-cultural validity), criterion validity and responsiveness. To complete the COSMIN checklist, we will follow a four-step procedure $^{53}$ :

1. Determine what measurement properties are evaluated in the article.

2. If the statistical methods described in the article are based on item response theory (IRT), determine whether the article meets the specified requirements for IRT.

3. Evaluate the article's methodological quality with regard to the properties identified in step 1.

4. Assess the generalisability of the results with regard to the properties identified in step 1.

The domains of the COSMIN checklist contain 5-18 items and each item can be rated as 'poor', 'fair', 'good' or 'excellent'. 5354 To allocate an overall methodological quality score for each article, we will use the scoring system proposed by the authors of the COSMIN checklist. ${ }^{54}$ Terwee et $a l^{54}$ suggest using the "worst score counts'principle, which means taking the lowest rating of any item in a checklist domain as the final quality rating for that domain. 


\section{Quality assessment of included instruments}

Based on the quality criteria for measurement properties proposed by Terwee $e t a l,{ }^{55}$ the measurement properties reported in the included articles will be rated as positive, negative or indeterminate, depending on the study design, methods and outcomes (table 3). For the proposed review, we will present an overview of these ratings in a table. Although the aforementioned criteria were developed for health status questionnaires, they can also be applied to performance-based assessments and other measurement methods. ${ }^{50}$

\section{Data synthesis}

If more than one article is reported on the same assessment and they are homogeneous concerning study population, design, the language, the version of the used instrument and the form of administration, we will perform 'a best evidence synthesis' as proposed by van Tulder et $a \bar{l}^{\tilde{6}}$ (table 4). We will rate the level of best evidence as 'strong', 'moderate', 'limited', 'conflicting' or 'unknown'. This step should facilitate choosing a suitable assessment.

\section{Patient and public involvement}

This review will be based on previous published data and no patients or the public will be involved in this review.

\section{DISCUSSION}

The proposed review will evaluate available MI ability assessments and their measurement properties across four important disciplines: sports, psychology, medicine and education. There is a need to evaluate the existing assessments systematically across disciplines. This is especially true now for the following reasons:

1. The benefits and effectiveness of MI have been confirmed and this technique is increasingly applied in different disciplines. ${ }^{135-810-14}$

2. The effect of MI interventions depends on the individual's MI ability and it should be evaluated prior to an MI intervention. ${ }^{14} 30$

3 . For the last 200 years, experts focused on developing assessments to evaluate MI's abstract construct. ${ }^{31-43}$ For these reasons, a systematic evaluation is warranted.

Collecting MI assessments from different disciplines allows and facilitates their cross-disciplinary usage and research. It is assumed that several MI assessment instruments will be attributed to more than one discipline. However, based on our experience, it is difficult for a hemiparetic patient to perform and imagine running or jumping during a MI ability assessment, as is sometimes suggested. Discipline-specific MI assessment might be more sensitive in some cases and thus, it is best not to focus on a movement that cannot be performed at the moment.

This systematic overview could help to select the most suitable MI ability assessment for the treatment aim and population and further evaluate the efficacy of MI training interventions. To date, only a few studies have offered a comprehensive evaluation of the methods by which MI ability has been assessed, much less these methods' psychometric properties. So far, there have been only a few studies on the assessment methods of MI ability, ${ }^{2} 178$ and for the studies that do exist, their overview is quite narrow and focused on population and language. Only two reviews evaluate the psychometric properties of the included tools ${ }^{178}$ and none of the cited reviews consider whether the MI ability assessments are appropriate for children. Furthermore, in a comprehensive review, Schuster et al reported that some of the included studies used individual, custom-designed assessments of MI ability, which limits the comparison of results with other studies.

\section{Presenting the review results}

Results of this review will be reported following the PRISMA guidelines, using flowcharts and tables. ${ }^{57}$ Information on assessing performance and equipment as well as presenting the included articles' methodological quality and the included instrument's quality assessments will offer athletes, trainers, clinicians, teachers and other interested MI user the much-needed tools to quickly determine an MI ability assessment's focus and its applicability and utility.

Acknowledgements We would like to thank Dr Michael A McCaskey for valuable feedback on a previous version of the manuscript.

Contributors ZS, PP-W, SK, AS-T, TE and CS-A contributed to the conception and the design of the protocol of the systematic literature review. All contributed important intellectual content, revised the protocol critically and agreed to publish the manuscript.

Funding The authors have not declared a specific grant for this research from any funding agency in the public, commercial or not-for-profit sectors.

Competing interests None declared.

Patient consent Not required.

Provenance and peer review Not commissioned; externally peer reviewed.

Open access This is an open access article distributed in accordance with the Creative Commons Attribution Non Commercial (CC BY-NC 4.0) license, which permits others to distribute, remix, adapt, build upon this work non-commercially, and license their derivative works on different terms, provided the original work is properly cited, appropriate credit is given, any changes made indicated, and the use is non-commercial. See: http://creativecommons.org/licenses/by-nc/4.0/.

\section{REFERENCES}

1. Schuster $\mathrm{C}$, Hilfiker $\mathrm{R}, \mathrm{Amft} \mathrm{O}$, et al. Best practice for motor imagery: a systematic literature review on motor imagery training elements in five different disciplines. BMC Med 2011;9:75.

2. Di Rienzo F, Collet C, Hoyek N, et al. Impact of neurologic deficits on motor imagery: a systematic review of clinical evaluations. Neuropsychol Rev 2014;24:116-47.

3. Braun S, Kleynen M, van Heel T, et al. The effects of mental practice in neurological rehabilitation; a systematic review and meta-analysis. Front Hum Neurosci 2013;7:390.

4. Zimmermann-Schlatter A, Schuster C, Puhan MA, et al. Efficacy of motor imagery in post-stroke rehabilitation: a systematic review. $J$ Neuroeng Rehabil 2008;5:8.

5. Cramer SC, Orr EL, Cohen MJ, et al. Effects of motor imagery training after chronic, complete spinal cord injury. Exp Brain Res 2007;177:233-42.

6. Lebon F, Guillot A, Collet C. Increased muscle activation following motor imagery during the rehabilitation of the anterior cruciate ligament. Appl Psychophysiol Biofeedback 2012;37:45-51. 
7. Marusic U, Grosprêtre S, Paravlic A, et al. Motor imagery during action observation of locomotor tasks improves rehabilitation outcome in older adults after total hip arthroplasty. Neural Plast 2018;2018:1-9.

8. Cupal DD, Brewer BW. Effects of relaxation and guided imagery on knee strength, reinjury anxiety, and pain following anterior cruciate ligament reconstruction. Rehabil Psychol 2001;46:28-43.

9. Christakou A, Zervas $Y$, Lavallee $D$. The adjunctive role of imagery on the functional rehabilitation of a grade II ankle sprain. Hum Mov Sci 2007;26:141-54.

10. Sordoni $C$, Hall $C$, Forwell $L$. The use of imagery by athletes during injury rehabilitation. J Sport Rehabil 2000;9:329-38.

11 Bucher $L$. The effects of imagery abilities and mental rehearsal on learning a nursing skill. J Nurs Educ 1993;32:318-24.

12 Doheny MOB. Effects of mental practice on psychomotor skills with baccalaureate nursing students. Dis Abstr Int 1990;51(2-B):659.

13 Immenroth M, Bürger T, Brenner J, et al. Mental training in surgical education: a randomized controlled trial. Ann Surg 2007;245:385-91.

14 Cumming J, Ramsey R. Imagery interventions in sport. 2009.

15. Jeannerod M, Frak V. Mental imaging of motor activity in humans. Curr Opin Neurobiol 1999;9:735-9.

16. de Vries S, Tepper M, Otten B, et al. Recovery of motor imagery ability in stroke patients. Rehabil Res Pract 2011;2011:1-9.

17. McAvinue LP, Robertson IH. Measuring motor imagery ability: a review. Eur J Cogn Psychol 2008;20:232-51.

18. Guillot $A$, Collet $C$, Nguyen VA, et al. Brain activity during visual versus kinesthetic imagery: an fMRI study. Hum Brain Mapp 2009;30:2157-72.

19. Callow N, Roberts R, Hardy L, et al. Performance improvements from imagery: evidence that internal visual imagery is superior to external visual imagery for slalom performance. Front Hum Neurosci 2013;7:697

20. Wakefield C, Smith D. Perfecting practice: applying the PETTLEP model of motor imagery. J Sport Psychol Action 2012;3:1-11.

21. Decety J, Perani D, Jeannerod M, et al. Mapping motor representations with positron emission tomography. Nature 1994;371:600-2.

22. Decety J. The neurophysiological basis of motor imagery. Behav Brain Res 1996;77(1-2):45-52.

23. Roth M, Decety J, Raybaudi M, et al. Possible involvement of primary motor cortex in mentally simulated movement: a functional magnetic resonance imaging study. Neuroreport 1996;7:1280-4.

24. Grosprêtre S, Lebon F, Papaxanthis C, et al. New evidence of corticospinal network modulation induced by motor imagery. $J$ Neurophysiol 2016;115:1279-88.

25. Sharma N, Baron JC. Does motor imagery share neural networks with executed movement: a multivariate fMRI analysis. Front Hum Neurosci 2013;7:564.

26. Paravlic AH, Slimani M, Tod D, et al. Effects and dose-response relationships of motor imagery practice on strength development in healthy adult populations: a systematic review and meta-analysis. Sports Med 2018;48:1165-87.

27. Lequerica A, Rapport L, Axelrod BN, et al. Subjective and objective assessment methods of mental imagery control: construct validation of self-report measures. J Clin Exp Neuropsychol 2002;24:1103-16.

28. Galton F. Inquiries into human faculty and its development: Macmillan, 1883.

29. Hall CR. Individual differences in the mental practice and imagery of motor skill performance. Can J App/ Sport Sci 1985;10:17-21.

30. Martin KA, Moritz SE, Hall CR. Imagery use in sport: a literature review and applied model. Sport Psychol 1999;13:245-68.

31. Galton F. Statistics of mental imagery mind. , 1880:os-V, 301-18.

32. Betts GH. The distribution and functions of mental imagery. New York, Teachers College: Columbia University, 1909:112.

33. Marks DF. Visual imagery differences in the recall of pictures. $\mathrm{Br} \mathrm{J}$ Psychol 1973;64:17-24

34. Isaac A, Marks DF, Russell DG. An instrument for assessing imagery of movement: the Vividness of Movement Imagery Questionnaire (VMIQ). J of Ment Imagery 1986;10:23-30.
35. Sheehan PW. A shortened form of Betts' questionnaire upon mental imagery. J Clin Psychol 1967;23:386-9.

36. Kwekkeboom KL. Measuring imaging ability: psychometric testing of the imaging ability questionnaire. Res Nurs Health 2000;23:301-9.

37. Blajenkova O, Kozhevnikov M, Motes MA. Object-spatial imagery: a new self-report imagery questionnaire. Appl Cogn Psychol 2006;20:239-63.

38. Malouin F, Richards CL, Jackson PL, et al. The Kinesthetic and Visual Imagery Questionnaire (KVIQ) for assessing motor imagery in persons with physical disabilities: a reliability and construct validity study. J Neurol Phys Ther 2007;31:20-9.

39. Roberts R, Callow N, Hardy L, et al. Movement imagery ability: development and assessment of a revised version of the vividness of movement imagery questionnaire. J Sport Exerc Psychol 2008:30:200-21

40. Hall C, Pongrac J, Buckholz E. The measurement of imagery ability. Hum Mov Sci 1985;4:107-18.

41. Hall CR, Martin KA. Measuring movement imagery abilities: a revision of the movement Imagery Questionnaire. J of Ment Imagery 1997;21(1-2):143-54.

42. Sharma N, Pomeroy VM, Baron JC. Motor imagery: a backdoor to the motor system after stroke? Stroke 2006;37:1941-52.

43. Malouin F, Richards CL, Durand A, et al. Clinical assessment of motor imagery after stroke. Neurorehabil Neural Repair 2008;22:330-40.

44. Switras JE. An alternate-form instrument to assess vividness and controllability of mental imagery in seven modalities. Percept Mot Skills 1978;46:379-84.

45. Collet C, Guillot A, Lebon F, et al. Measuring motor imagery using psychometric, behavioral, and psychophysiological tools. Exerc Sport Sci Rev 2011;39:85-92.

46. Dettmers $\mathrm{C}$, Benz M, Liepert $\mathrm{J}$, et al. Motor imagery in stroke patients, or plegic patients with spinal cord or peripheral diseases. Acta Neurol Scand 2012;126:238-47.

47. Heremans $E$, Feys $P$, Nieuwboer $A$, et al. Motor imagery ability in patients with early- and mid-stage Parkinson disease. Neurorehabil Neural Repair 2011;25:168-77.

48. Melogno-Klinkas M, Nunez-Nagy S, Ubillos S. Outcome measures on motor imagery ability:use in neurorehabilitation. Maastricht, Netherlands: The 2nd International Congress on Neurorehabilitation and Neural Repair, 2017.

49. Shamseer L, Moher D, Clarke M, et al. Preferred reporting items for systematic review and meta-analysis protocols (PRISMA-P) 2015: elaboration and explanation. BMJ 2015;349:g7647.

50. de Vet HCW, Terwee CB, Mokkink LB, et al. Measurement in medicine: a practical guide. Cambridge University Press, 2011.

51. Terwee CB, Jansma EP, Riphagen II, et al. Development of a methodological PubMed search filter for finding studies on measurement properties of measurement instruments. Qual Life Res 2009;18:1115-23.

52. Cohen J. A coefficient of agreement for nominal scales. Educ Psychol Meas 1960;20:37-46.

53. Mokkink LB, Terwee CB, Patrick DL, et al. The COSMIN checklist for assessing the methodological quality of studies on measurement properties of health status measurement instruments: an international Delphi study. Qual Life Res 2010;19:539-49.

54. Terwee CB, Mokkink LB, Knol DL, et al. Rating the methodological quality in systematic reviews of studies on measurement properties: a scoring system for the COSMIN checklist. Qual Life Res 2012;21:651-7

55. Terwee CB, Bot SD, de Boer MR, et al. Quality criteria were proposed for measurement properties of health status questionnaires. $J$ Clin Epidemiol 2007;60:34-42.

56. van Tulder M, Furlan A, Bombardier C, et al. Updated method guidelines for systematic reviews in the cochrane collaboration back review group. Spine 2003;28:1290-9.

57. Moher D, Liberati A, Tetzlaff $\mathrm{J}$, et al. Preferred reporting items for systematic reviews and meta-analyses: the PRISMA statement. PLOS Med 2009;6:e1000097. 